DOI: https://doi.org/10.47631/jsrmbs.v2i3.242

\title{
Polymyxins: "Last Resort” for MDR and/or XDR Gram-Negative Infections
}

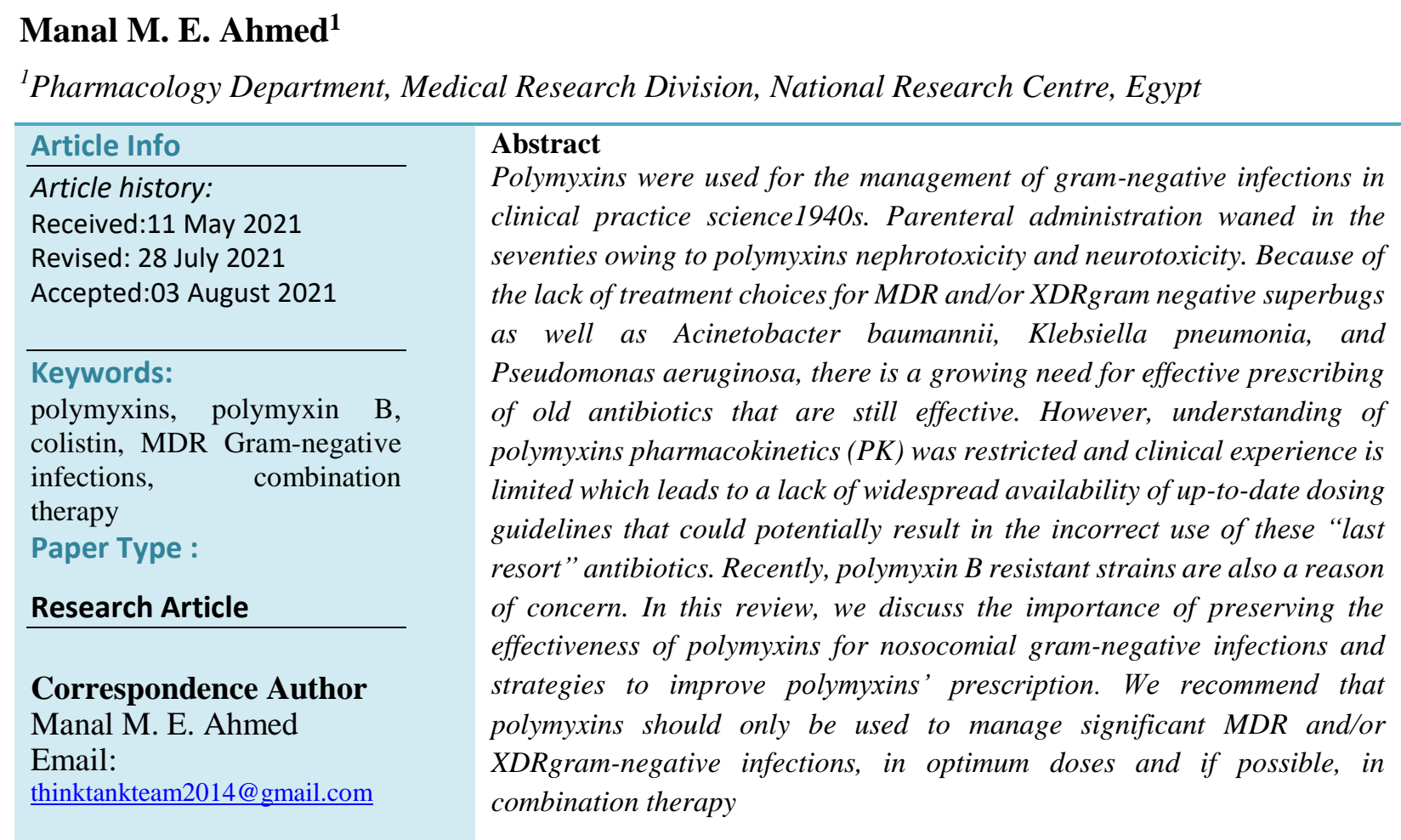

\section{Introduction}

Microbial resistance to antibiotics has a growing interest as it represents a vital issue for public health (Ventola, 2015; Hooton \& Levy 2001). The resistance of Gram-negative bacteria (GNB) is of special concern for both bacteriologists and clinicians because of the fastspreading of antibiotics resistance and the very limited treatment options (Shaikh et al., 2015). It is worthy to mention that the fast spread of antibiotics resistance is not matched by the creation of novel promising molecules of antibiotics (Hooton \& Levy, 2001). Therefore, there is a growing need for effective prescribing of old antibiotics that are still effective against multidrug and extremely drug-resistant (MDR and XDR) bacteria. International collaborative efforts are called to achieve this goal. In this concern, polymyxins is one of the frontline antibiotics which have not been used widely in the previous years (Theuretzbacher et al., 2015). In the 1940s, polymyxins were approved for clinical use, while by the early 1970s, their severe side effects including severe nephrotoxicity and neurotoxicity limited their usage (Falagas et $a l ., 2005)$. Because of the lack of use in the last 50 years, pharmacodynamics (PD) of polymyxins is very limited. Recently, polymyxins have regained significant interest. Intravenous (iv) administration of these drugs has substantially increased in the last 
decade due to their response in several infections caused by GNB especially the MDR bacteria such as Pseudomonas aeruginosa, Acinetobacter baumannii, and Klebsiella pneumoniae has been significant (Gupta et al., 2009). Growing usage of polymyxins in GNB infections was recognized and perceived in consensus for maximizing the clinical use of polymyxins "The Prato Polymyxin Consensus" (Nation et al., 2015). Given the increasing use of polymyxin B in clinical settings. In this review, we discuss current usages of polymyxin B as monotherapy and in combinations and highlight the urgency of obtaining knowledge on their pharmacology to optimize their clinical usage and minimize the potential for bacterial resistance development.

\section{Chemical structure of Polymyxins}

Polymyxins group including polymyxin B and colistin (polymyxin E) is the "old" antibiotics that are clinically used. There has been a revived concern in this group of antibiotics because of their widespread resistance to newer antibiotics. Polymyxins are now labeled as (last resort" for MDR and/or XDR Gram-negative infections) (Kwa et al., 2008). Although polymyxins were approved for clinical usage in the 1940s, they were not favored referring to their toxicities. However, their growing usage in critical care settings has helped for understanding their behavior in both Vitro and Vivo (Kwa et al., 2008). The composition of polymyxins is a fatty acid chain (hydrophobic region) and amino acids ( $\mathrm{D}$ and $\mathrm{L}$ ) arranged in a cyclical heptapeptide ring. A tripeptide side chain binds the cyclical ring to the fatty acid chain. The single amino acid chain of D-leucine in polymyxin $\mathrm{E}$ is replaced by $\mathrm{D}$-phenylalanine in polymyxin B (Figure 1) (Kwa et al., 2008; Zavascki et al., 2007; Landman et al., 2008). The commercial form of polymyxin B is available as sulphate salt for parenteral administration (Kwa et al., 2008). Different polypeptide components in polymyxin B including B1, B2, B3, and B1-I have different molecular formulas and the sum of these constitutes a minimum of $80 \%$ for polymyxin B. In concern of these components, there is batch to batch variation in commercial preparations (Kwa et al., 2008).

The difference between polymyxin B and colistin is at R6. It is D-phenylalanine in polymyxin B and D-leucine in colistin. Forcolistinmethanesulphonate, there is the addition of a sulphomethyl group to the primary amines of colistin resulted in a change in the electrostatic charges. Thr: threonine; Leu: leucine; Phe: phenylalanine; Dab: diaminobutyric acid. CMS: colistin methanesulphonate (Deris et al. 2014).

\section{Polymyxins mechanism of Action}

Polymyxins' antimicrobial effect can be achieved by two mechanisms. Firstly, being positively charged, these cationic polypeptides interact electrostatically with bacterial lipopolysaccharide (LPS) that presents in GNB outer cell membrane. These interactions displace positively charged $\mathrm{Ca}++$ and $\mathrm{Mg}++$ (stabilizers of lipopolysaccharide in the outer cell membrane). This leads to instability of cell membrane-like detergent effect resulting in leakage of cell contents and accelerating bacterial death. Secondly, polymyxins have potent anti-endotoxin activity. Polymyxinsbinding to lipid A that is a component of LPS molecules neutralizing it. Whether the mechanism by which septic shock prevention occurs has not been understood yet. It is thought that plasma endotoxin is immediately bound by LPS-binding protein, and the complex is quickly bound to cell-surface CD14 (Gupta et al., 2009; Kwa et al., 
2008). Another mechanism of bacterial killing by polymyxins involves inhibition of protein function has also been investigated. Deris et al., (2014) explored that the type-II NADHquinone oxidoreductase (NDH-2) is inhibited by polymyxin B as shown in figure (2) thus ubiquinone binding was competitively inhibited, and NADH was non-competitively inhibited by polymyxin B.Thesame finding has been proved in Mycobacterium tuberculosis. Despite the specific details of polymyxin-inducing bacterial killing still unknown, the primary interaction between polymyxins with lipid A is pivotal to the killing process; this is exemplified in the currently recognized resistance mechanisms.

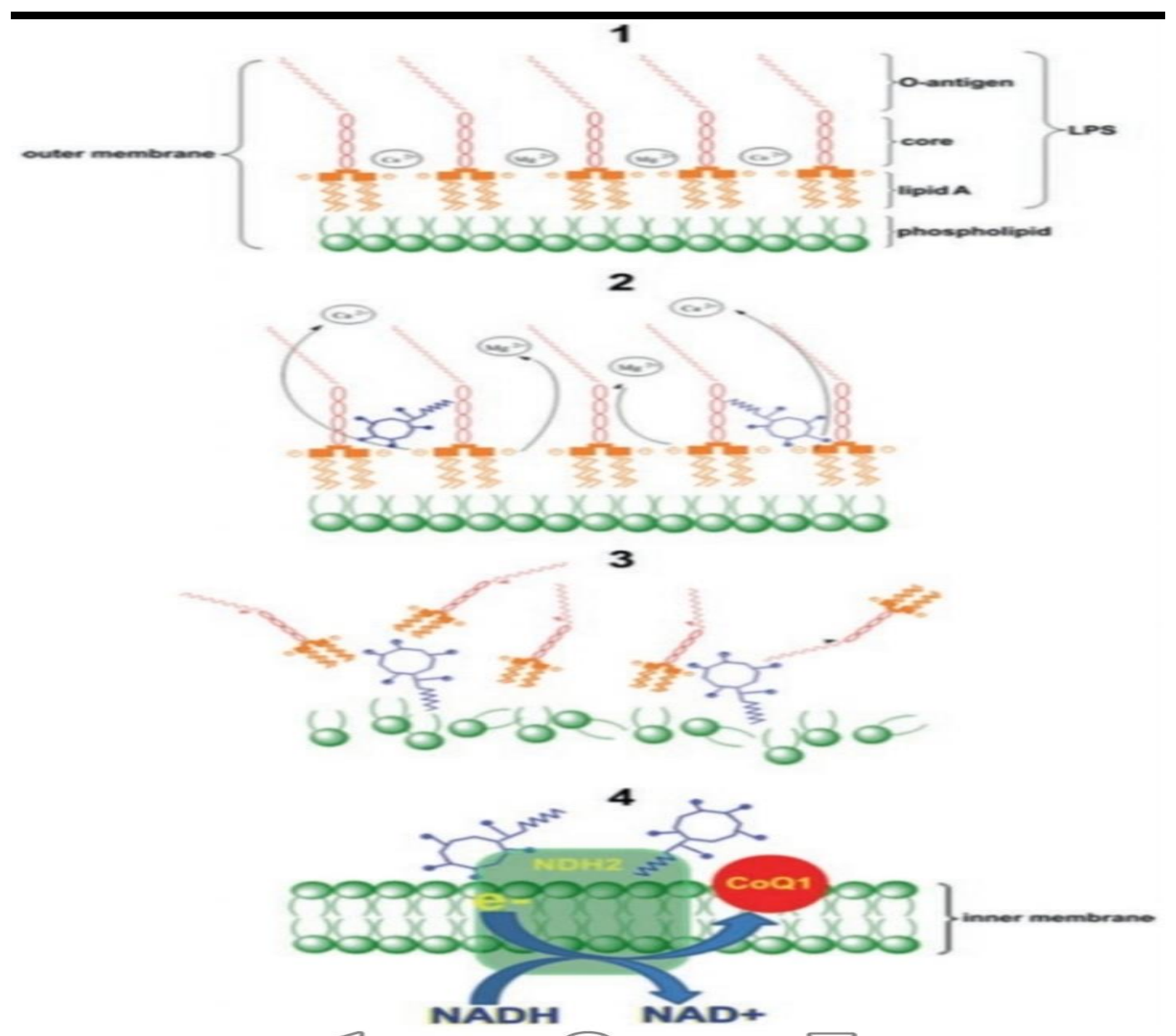

Figure 2: Diagrammatic representation for polymyxins mechanism of action. Figure is adapted from Deris et al.,2014.

1) Polymyxins target the outer membrane of Gramnegative bacteria.

2) The positively charged polymyxins displace divalent cations that bridge adjacent LPS molecules.

3) The electrostatic interaction weakens the stability of the outer membrane and the hydrophobic insertion destabilizes the outer membrane through hydrophobic expansion resulting in damage to the outer membrane.

4) Polymyxins penetrate the inner membrane and inhibit the respiratory enzyme NDH-2.

\section{The spectrum of polymyxins:}


The activity of polymyxin B is bactericidal mainly against Gram-negative bacteria such as Enterobacter spp., Pseudomonas aeruginosa, Acinetobacter spp. Escherichia coli, Salmonella spp., Klebsiella, Shigella spp., Citrobacter spp., Yersinia pseudotuberculosis, Haemophilusinfluenzae, Pasteurellaspp., Bordetella pertussis, and Legionella pneumophila. Thus we can say that most nosocomial infections are susceptible to polymyxins (Kwa et al., 2008; Landman et al., 2008). However, some Gram-negative isolates are intrinsically resistant to polymyxinslikeBurkholderia spp., Proteuss pp., Providencia spp., Morganellamorganii, and Serratia spp. Also, Brucella spp., Neisseria spp., and Chromobacterium spp. isolates are resistant. Both of Grampositive bacteria and anaerobes are resistant to polymyxins $[8,10]$. While the minimal inhibitory concentrations (MIC50 and MIC90) are varied according to species of bacteria. For major susceptible GNB, MIC50 and MIC90 were $\leq 1$ and $2 \mathrm{mg} / \mathrm{L}$ while for Acinetobacter spp., $\leq 1$ and $>8 \mathrm{mg} / \mathrm{L}$ for Aeromonas spp., $\leq 1$ and $2 \mathrm{mg} / \mathrm{L}$ for $P$. aeruginosa , $\leq 1$ and $\leq 1 \mathrm{mg} / \mathrm{L}$ for E. coli, and $\leq 1$ and $\leq 1 \mathrm{mg} / \mathrm{L}$ for Klebsiella spp. Respectively (Zavascki et al., 2007). MIC90 for most isolates of B. cepacia, S. maltophilia, Proteusspp., Proteus mirabilis, Serratiaspp., and other enteric GNB was more than $8 \mathrm{mg} / \mathrm{L}$ because of the intrinsic resistance in these bacterial isolates (Zavascki et al., 2007).

\section{Resistance Mechanisms for polymyxins}

Susceptibility breakpoints 2007, Clinical and Laboratory Standards Institute (CLSI) performed the susceptibility testing of polymyxins. For Pseudomonas aeruginosa, Acinetobacter, and Enterobacteriaceae, susceptibility breakpoints were as shown in table (1) (Zavascki et al., 2007).

\begin{tabular}{|c|c|c|c|}
\hline \multirow{2}{*}{ Organism } & \multicolumn{3}{|c|}{ Profile } \\
\hline & Susceptible & Intermediate & Resistant \\
\hline \multicolumn{4}{|l|}{ CLSI recommendations [18] } \\
\hline P. aeruginosa" & $\mathrm{MIC} \leq 2 \mathrm{mg} / \mathrm{L}$ & $\mathrm{MIC}=4$ & $\mathrm{MIC} \geq 8 \mathrm{mg} / \mathrm{L}$ \\
\hline Acinetobacter spp." & $\mathrm{MIC} \leq 2 \mathrm{mg} / \mathrm{L}$ & - & $\mathrm{MIC} \geq 4 \mathrm{mg} / \mathrm{L}$ \\
\hline Non-Enterobacteriaceae* & $\mathrm{MIC} \leq 2 \mathrm{mg} / \mathrm{L}$ & $\mathrm{MIC}=4$ & $\mathrm{MIC} \geq 8 \mathrm{mg} / \mathrm{L}$ \\
\hline \multicolumn{4}{|l|}{ BSAC Recommendations [19] } \\
\hline Pseudomonas spp." & $\mathrm{MIC} \leq 4 \mathrm{mg} / \mathrm{L}$ & - & $\mathrm{MIC} \geq 8 \mathrm{mg} / \mathrm{L}$ \\
\hline Enterobacteriaceae spp." & $\mathrm{MIC} \leq 4 \mathrm{mg} / \mathrm{L}$ & - & $\mathrm{MIC} \geq 8 \mathrm{mg} / \mathrm{L}$ \\
\hline
\end{tabular}

\section{The mechanisms}

The mechanism of resistance involving preliminary alterations in interaction between polymyxin with bacterial LPS. Intrinsically resistant isolates of Proteus mirabilis, Burkholderiacepacia, and Chromo bacterium violaceum developed modification in lipid A component of LPS in outer cell membrane resulting in a decrease in binding of polymyxins. The major modification noticed in LPS is that the 4-phosphate moiety of LPS is linked to 4amino-4-deoxy-L-arabinopyranose making isolates resistant to polymyxins. Acquired resistance in Salmonella spp. and E. coli associated with reduced susceptibility to polymyxins 
because of lipid A modification. Lipid A alteration with 4-amino-4-deoxy-L-arabinose (LAra4N) and/or phosphoethanolamine (PEtn) tends to reduce LOS negative charge which leads to reduced binding and increased resistance to polymyxins. Also, $K$. pneumoniae capsule is reported to be a potential factor for driving resistance (Zavascki et al., 2007; Landman et al., 2008). Additionally, in vitro, conditions of culture medium are found to be responsible for resistance to polymyxins (McLeod, G. I., \& Spector, M. P. (1996).

\section{Polymyxins pharmacokinetics}

Althoughpolymyxins have been applied clinically, understanding pharmacokinetics (PK) was only restricted for polymyxin B. Recently, the details of PK of polymyxins have been studied. Kwa et al. studied the PK of polymyxin B in MDRGram-negative infections in adults $\geq 16$ years without renal dysfunction. In a dose of 0.3 to 1 million units administered once or twice daily for a mean duration of 7 days, the reported mean volume of distribution $(\mathrm{Vd})$ was $42.7 \mathrm{~L}$ with a half-life of 13.6 hours. Mean clearance was $2.4 \mathrm{~L} / \mathrm{h}$. This study leads to understanding PK of polymyxin B but its limitation was the small number $(n=9)$ of subjects (Kwa et al., 2008). It was thought that polymyxin B doses should be adjusted according to the renal function (Gupta et al., 2009) but a recent study by Sandri et al. (2013) provided enough details of population PK of polymyxin B in critically ill patients. Twenty-four patients (above 20 years) who had varying creatinine clearance administrated polymyxin B in a dose of $0.45-$ $3.38 \mathrm{mg} / \mathrm{kg} / \mathrm{day}$. They showed significantly low inter-individual variability in total body clearance when the dose was scaled by total body weight and not by total creatinine clearance. The coefficient of variation was $32.4 \%$. Therefore, the authors concluded that polymyxin B clearance did not demonstrate any correlation with creatinine clearance even in patients who were on renal replacement therapy. They suggested that polymyxin B does not require modification of dose even in patients on renal replacement therapy. A large amount of filtered polymyxin Bis reabsorbed from kidneys in a linear relationship with creatinine clearance suggesting higher reabsorption with declining renal function.

Furthermore, this study highlighted the dosing of polymyxin B according to MICs of causative organism, a high dose regimen ( $3 \mathrm{mg} / \mathrm{kg} / \mathrm{d})$ is necessary for $\mathrm{MIC} \leq 2 \mathrm{mg} / \mathrm{L}$ wherein loading dose should be considered. In less severe infection, for $\mathrm{MIC} \leq 1 \mathrm{mg} / \mathrm{L}$, a usual dose of up to $2.5 \mathrm{mg} / \mathrm{kg} / \mathrm{d}$ would be appropriate. For higher MICs, the dose must not exceed $3 \mathrm{mg} / \mathrm{kg} / \mathrm{d}$ for safety concerns (Sandri et al., 2013). In another small trial of 8 patients, PK data about polymyxin B revealed a peak plasma concentration of 2.38 to $13.9 \mathrm{mg} / \mathrm{L}$ at the end of a 1-hour intravenous (IV) infusion. Unchanged drugrecoveryinurinewas $0.04 \%-0.86 \%$ of the dose. Other further studies proved that polymyxin B clearance is independent of renal function and is eliminated mainly by nonrenal pathways (Zavascki et al., 2008).

\section{Pharmacodynamics}

Studies of polymyxin B based on the time-kill against different isolates of $P$. aeruginosa, K. pneumoniae, and A. baumannii showed concentration-dependent killing 
(Landman et al., 2008), which was followed by the regrowth of the isolates. These isolates were recorded to have higher MICs for polymyxin B.P. aeruginosa in vitro study with dosing interval in between 12 and 24 hours was associated with the emergence of resistant isolates comparing with shorter interval dosing (Tam et al., 2005; Bergen et al., 2008).

For this resistant development and reduction in susceptibility of such isolates, it is advisable to prescribe polymyxins in combination therapy. While usage of polymyxins in combinations in vitro studies has been reported to be associated with a reduction in regrowth of isolates, reduction in polymyxin B resistance and bactericidal activity even at sub-MIC concentrations of polymyxins. Although the evidence with polymyxins combinations clinically is still quite limited (Kwa et al., 2008; Landman et al., 2008).

\section{Polymyxin B as Monotherapy}

As described above, polymyxin B is composed of polypeptide components. These components were evaluated in vitro against three standard wild-type bacterial strains and three clinical MDR strains of $P$. aeruginosa, A. baumannii, and K. pneumoniae. Determination of MIC was performed using the dilution method in both. For potency, no substantial variations were noticed against standard and MDR strains suggesting that antibacterial activity has not been affected by molecular structure (Tam et al., 2011). Alsoassessmentof polymyxin B activity against carbapenem-resistant $A$. baumannii (CRAB) was performed by another study (Thamlikitkul et al., (2014). For 217 clinical strains of CRAB, MIC50 and MIC90 values were 0.5 and $1 \mathrm{mg} / \mathrm{L}$, respectively. With a breakpoint of $\leq 2 \mathrm{mg} / \mathrm{L}, 98.2 \%$ of strains were identified to be susceptible. These findings proved the efficacy of polymyxin B for CRAB infections. These results were supported by another Mexico study (Rosales-Reyes et al., 2016) which demonstrated $100 \%$ susceptibility to highly lethal (mortality rate up to $28.2 \%$ ) and biofilmproducing colonies (92.9\% strains) - MDR A. baumannii - to polymyxin. Those were resistant to most antibiotics including aminoglycosides, cephems, carbapenems, and fluoroquinolones. Thus we can conclude the superiority of polymyxin B efficacy against MDR and biofilmproducing A.Baumanniiisolates.

From all of the above, we can consider polymyxin B as the last resort in MDR Gramnegative infections. In a retrospective analysis of critically ill children $(\leq 15$ years) with MDR Gram-negative infections ( $\mathrm{n}=14$ ), polymyxin B administered in a dose of 40,000 IU/kg/day resulted in the survival of $57.1 \%$ of children. In addition, many clinical trials have been reported that the sensitivity of Acinetobacterspp., P. aeruginosa, K. pneumoniae, and Enterobacter spp. isolates reached up to $100 \%$ while nephrotoxicity was evident in three cases (Qamar et al., 2014). Thus, polymyxin B was found as the modality to treat MDR Gram-negative infections. This calls for judicial use of polymyxins in critical cases. Kvitko et al. (2011) performed a retrospective evaluation for IV polymyxin Befficacy with a mean dose of $141 \pm 54 \mathrm{mg}$, twice daily comparing to other antibiotics in patients with $P$. aeruginosa bacteraemia. In 133 patients (33.8\% with polymyxin B and $66.2 \%$ with others; most common being beta-lactams (83\%)), in-hospital mortality was observed to be significantly higher $(\mathrm{p} \leq 0.001)$ with polymyxin $\mathrm{B}$ $(66.7 \%)$ than comparators $(28.4 \%)$. Though mortality was higher with polymyxin B, optimize dosage utilization is crucial to reduce such outcomes. For preserving polymyxin B efficacy to susceptible strains, priority must be undertaken for decreasing or preventing the emergence of 
resistance. Nelson et al (2015) Retrospective study evaluated the efficacy of polymyxin B for managing bloodstream infections caused by carbapenem-resistant Gram-negative rods ( $\mathrm{n}=$ 151, K. pneumoniae $60.9 \%$, A. baumannii $21.2 \%$, and $P$. aeruginosa $11.3 \%$ ). Overall 30-day mortality was $37.8 \% .63 .6 \%$ were found to have a clinical cure at day 7 of treatment. Post analysis shown a significant higher mortality with dose $<1.3 \mathrm{mg} / \mathrm{kg} / \mathrm{day}(\mathrm{n}=0.02)$ but no difference observed in clinical cure at day $7(\mathrm{n}=0.70)$. With a dose of $250 \mathrm{mg} / \mathrm{d}$ or more, acute kidney injury was noticed to be significantly greater $(n=0.03)$ which persisted in a multivariable analysis (odds ratio (OR) 4.32; $\mathrm{n}=0.03$ ).

In another similar study, Elias et al. (2010) concluded with the impact of polymyxin B

dose on mortality outcome. In this retrospective assessment, patients $(\mathrm{N}=276)$ receiving polymyxin B for over 72 hours were included and subgroup microbiological analysis confirmed infections and those with bacteraemia. The overall mortality rate was $60.5 \%$. Septic shock (adjusted OR (a OR) 4.07), use of mechanical ventilation (aOR 3.14), Charlson comorbidity score (aOR 1.25), and age (aOR 1.02) were independent predictors of mortality. Polymyxin B in a dose of $200 \mathrm{mg} /$ day or above was associated with significantly lower mortality outcomes (aOR 0.43) and this effect was the same in both subgroups. On the other hand, this dose had a higher risk of severe renal impairment. These findings highlight the fact that a higher dosage of polymyxin B benefits in terms of reducing in-hospital mortality. This association needs further exploration in a large, prospective, randomized trial. Increased risk of renal injury calls for a careful look at coexisting factors that might contribute to renal damage. Therefore, targeting these factors may provide benefits in reducing the severity of renal injury accompanied with polymyxin Badministratio. The risk factors associated with polymyxin B monotherapy treatment failure in clinical cases $(n=40)$ of carbapenem-resistant K. pneumonia (CRKP) were retrospectively assessed by Dubrovskaya et al. (2013).

Clinical and microbiological cures were reported in 73\%and 53cases, respectively. Overall, the 30 -day mortality reported was $28 \%$. After adjusting for septic shock, baseline renal insufficiency was observed to be associated with a 6 times greater chance of clinical failure. Additionally, the observation of some breakthrough infections was intrinsically resistant to polymyxin B. Therefore, the leading cause of polymyxins failure as monotherapy may be regarding baseline renal dysfunction and subsequent development of resistant infections. Improving the efficacy and preventing the emergence of resistance in polymyxin B may be achieved by prescribing it in combination with other antibiotics.

\section{Polymyxin B as Combination Therapy}

It is recommended to use combination therapies for managing MDR and extremely drug-resistant (XDR) organisms including superbugs. In this scope of development of bacterial resistance, combination therapy with polymyxin B may be considered a promise for critically ill patients. Clinical usage of combination therapy may decrease the development of bacterial resistance in comparison to monotherapy (Bergen et al., 2015). Synergistic efficacy of polymyxinBandchloramphenicol in MDRNDM-producing $K$. pneumoniae was observed by (Rahim et al. 2015). Chloramphenicol alone was ineffective with these strains and polymyxin $\mathrm{B}$ as monotherapy was also associated with rapid regrowth and emergence of resistance. Using critical care research and practice 5 combinations, no polymyxin-resistant isolates were 
recorded. Scanning electron microscopy (SEM) features also were consistent with these findings. They found the formation of projections and blebs on the surface of bacterium which is consistent with the mechanism of polymyxin B and they were denser with combination treatment. This provides insights that combination treatment may avert the development of resistance to polymyxin B. This observation adds to the finding that antibiotics considered "old" can be beneficial even in superbug infections when used in combination.

One of the important nosocomial infections is carbapenem-resistant A. baumannii $(\mathrm{CRAB})$ as combination therapies may prove beneficial effects. Three antibiotics - polymyxin $\mathrm{B}$, rifampicin, and tigecycline were evaluated by Lim et al. (201) alone and in combination in such infections. Among 31 MDR isolates, allweresusceptibletopolymyxinBasmonotherapy. Time-kill studies, no antibiotic had bactericidal activity. In combination, polymyxin and rifampicin shown the highest bactericidal activity (41.9\%) followed by polymyxin and tigecycline (29.0\%) and tigecycline and rifampicin (22.6\%). Also, Hagihara et al. (2014) confirmed that polymyxin B and tigecycline $(200 \mathrm{mg})$ resulted in a significantly greater reduction in bacterial density and the area under bacterial killing and regrowth curve (AUBC) comparing to polymyxin Bmono therapy. Finally, the authors concluded that combination therapy is an effective tool for $\mathrm{CRAB}$ even with polymyxin B-sensitive infections.

Combination of minocycline and polymyxin $\mathrm{B}$ inA. Baumannii was evaluated by Bowers et al. (2015). The results revealed that polymyxin B improved intracellular penetration and thereby concentration of minocycline as well as enhanced in vitro bactericidal activity. This finding further proves the importance of combination treatment with polymyxin $\mathrm{B}$. In another trial, the activity of polymyxin B in combination with imipenem, meropenem, or tigecycline in KPC-2 producing Enterobacteriaceae was assessed by Barth et al. (2015). Six strains including K. Pneumonia $(\mathrm{n}=2)$,Enterobacter cloacae $(\mathrm{n}=2)$, andSerratiamarcescens $(\mathrm{n}$ $=2$ ) had decreased susceptibility or resistant to polymyxin B and/or tigecycline and resistant to carbapenems. Polymyxin B in combination with carbapenem was most effective against $K$. Pneumonia and Enterobacter cloacae in comparison to the tigecycline combination. For Serratiamarcescens, polymyxin B in combination with meropenem was highly effective and had synergistic bactericidal action.

In an extensively drug-resistant (XDR) A. baumannii(XDR-AB) study, polymyxin B in combination with imipenem, meropenem, doripenem, rifampicin, and tigecycline resulted in superior bactericidal activity compared to monotherapy. This suggests combination therapy must be considered in suspected XDR infections (Teo et al., 2015). Data about polymyxin combination therapy are available from small, retrospective, observational studies while clinical studies are limited. Large, prospective, randomized studies are highly important to prove the benefits and the recommended optimal dosage (Bergen et al., 2015). In an observational cohort study, polymyxin B in different combination therapies for carbapenemresistant Gram-negative bacteria was evaluated by Crusio et al. (2014). Various infections included $A$. baumannii(n=34/104), K. pneumonia(n=25/104), $P$. aeruginosa $(\mathrm{n}=11 / 104)$, and other multiple organisms ( $n=34 / 105)$. Five cases showed bacteremia. They summarized clinical and microbiological success, hospital mortality, and 6-month mortality in five groups. No significant differences were recorded between groups in-hospital mortality as well as in 6- 
month mortality outcome. Age, the severity of infection, and the Charlson score had a significant association with hospital mortality.

For XDRA. BaumanniiorP. aeruginosa clinical cases, Rigattoet al. (2015) reported a significantly lower rate of 30-day mortality in combination therapy in comparison to polymyxin $\mathrm{B}$ alone ( $42.4 \%$ versus $67.6 \%$, respectively, $\mathrm{n}=0.03$ ). Even in multivariate analysis, the combination treatment was reported to be independently associated with 30-day mortality. Specifically, the polymyxin B combination was useful with beta-lactams or carbapenems in $A$. Baumannii infections.P. aeruginosa associated mortality was significantly lower with the combination as compared to monotherapy $(\mathrm{n}=0.005)$. Generally, data confirmed the superior efficacy of polymyxin B-based combination therapy in treating MDR and XDR Gram-negative infections. In addition, the prescription of a validated polymyxin combination therapy based on multiple combination bactericidal testing was found superior to nonvalidated combination therapy and polymyxin monotherapy in decreasing mortality for cases suffering from XDR Gram-negative infections (Cai et al., 2016). While testing bactericidal activity of combination agents and thereafter combining these agents can reduce infection-related mortality, the empiric combination should not be delayed in a critical setting that can be further modified after sensitivity testing.

\section{Polymyxins synergy with antifungals}

Additionally, polymyxins are exhibiting weak fungicidal properties (MIC $=8 \mathrm{mg} / \mathrm{L}$ ). This synergistic antifungal activity of polymyxin B was studied as early as 1972. For example, polymyxin Bpotentiatesthe activity of tetracycline against Candida albicans and Saccharomyces cerevisiae, even at low concentrations. It seemed that polymyxins increase the permeability of the yeast Cell membrane to tetracycline, which inhibited protein synthesis resulting in cell death (Schwartz et al., 1972). A more recent study showed that combinations of polymyxin B with fluconazole or itraconazole are more effective even at low concentrations against Aspergillusfumigatus, Rhizopusoryzae, Candida albicans and non-albicans Candida species. These combinations at clinically relevant low concentrations were particularly potent against Cryptococcusneoformans, involving resistance strains to fluconazole (Zhai et al., 2010). Polymyxin B has also been decreased the tissue fungal burden both in intravenous and inhalation models of murine cryptococcosis at a level comparable to that of fluconazole (Zhai \& Lin, (2013).

Synergistic antifungal activity against $C$. albicans has also been achieved when both of polymyxin B and colistin were combined with amphotericin B, ketoconazole and miconazole against R. oryzae (Pietschmann et al., 2009). Both as a single agent and in combination with voriconazole, caspofungin and amphotericin $\mathrm{B}$, has also shown antifungal activity in vitro against filamentous ascomycetes causing cystic fibrosis in patients. This activity may provide a new therapeutic approach, especially for MDRS cedosporium prolificans (Schemuth et al., 2013).

Recently, susceptibility of 25clinical isolates of Fusarium to antifungal agents including amphotericin $B$, caspofungin, itraconazole, voriconazole, and antimicrobials pentamidine $\mathrm{B}$, tigecycline and tobramycin was evaluated in vitro. Amphotericin $\mathrm{B}$ or voriconazole in combination with tobramycin showed the highest rates of synergism $(80 \%$ and 
$76 \%$, respectively) followed by polymyxin B (76\% and 64\%) and pentamidine ( $72 \%$ and $68 \%$ ) (Venturini et al., 2016; Hsu et al., 2017). Also, caspofungin and echinocandin antifungals when combined with colistin have been shown to act synergistically against fluconazole-resistant and susceptible $C$. albicans and $C$. glabrata isolates. The authors also mentioned that the correlation with in vivo benefits may not be straightforward (Adams, et al., 2016; Pankey et al., 2014; Zeidler et al., 2013).

\section{Antibiofilm activity}

The biofilm is considered as an organized microbial ecosystem, consisting of one or more microbial species which are embedded in a self-produced matrix of extracellular polymeric substances that contain proteins, polysaccharides and DNA. Biofilms may appear on the body tissues as well as the surfaces of medical devices. Management of these biofilms mostly needs a high dose of antibiotics administrated for a long time. Polymyxins have been proven to be effective against biofilms, alone or in combination therapy specifically against $A$. baumannii and P. aeruginosa (Gopal et al., 2014; Lora-Tamayo et al., 2014). However, neither colistin nor polymyxin B were able to prevent (p)ppGppaccumulation (alarmones guanosine 5'-diphosphate 3'-diphosphate, ppGpp, and guanosine 5'-triphosphate 3'-diphosphate, pppGpp), signaling nucleotides that regulate the stringent response in bacteria and which are thought to play a vital role in the formation of biofilm (de la Fuente-Nu'nez et al., 2014).

Polymyxins were examined for showing an antibiofilm synergistic interaction with cyclic antimicrobial peptide gramicidin $\mathrm{S}$ toward 17 multidrug-resistant $P$. aeruginosa and biofilms of $P$. aeruginosa strain PAO1. The required concentration of PolymyxinBforinhibitionbiofilm formation by $P$. aeruginosa PAO1 was $8 \mathrm{mg} / \mathrm{mL}$ while treatment with gramicidin $\mathrm{S}$ as combination therapy required only $2 \mathrm{mg} / \mathrm{mL}$. Also, gramicidin S concentration was reduced from $32 \mathrm{mg} / \mathrm{mL}$ to $4 \mathrm{mg} / \mathrm{mL}$ in this combination. The fractional inhibitory concentration (FIC) calculated from this decrease was 0.375 , which indicated the synergistic effect of this treatment (Berditsch et al., 2015). Inhibition of biofilm formation by P.aeruginosa PAO1 strain has also been established. Antimicrobials that inhibit biofilm formation such as colistin and tobramycin, both alone and in combination, demonstrated bactericidal effect before biofilm attachment to endotracheal tubes while there is no activity was noticed once the biofilm formed on such polyvinylchloride tubes (Tarquinio et al., 2014). It is proved that polymyxin B is $100 \%$ effective in vitro against a highly prevalent clone of multi-drug resistant $A$. baumannii, with $92.9 \%$ of strains being biofilm producers. However, no direct proof of polymyxin inhibiting biofilm formation in this clone was recorded (R. Rosales-Reyes et al., 2015).

Recently, colistin entrapped in nanoparticles of different materials, [poly (lactide-coglycolide), chitosan and poly (vinyl alcohol)], has been found to eradicate pre-formed $P$. aeruginosa biofilms. Nanoparticles of colistin/poly (vinyl alcohol) and colistin/chitosan could penetrate inside the biofilms and release colistin in situ, thus increasing the effectiveness of the therapies (d'Angelo et al., (2015). An additive or synergistic effect between colistin and levofloxacin has been established in vitro and ina Galleria mellonella model against colistinsusceptible A.bumannii strains but not against colistin-resistant strains (Wei et al., 2015). 


\section{Polymyxins clinical Safety}

\section{Nephrotoxicity}

It is a well-known side effect of polymyxins. Most previous studies and case reports reported its high incidence of nephrotoxicity but with no specific definition of renal dysfunction. It was regarded mainly as intramuscular administration. Polymyxin B was identified to be associated with a higher incidence of renal toxicity comparing to colistin/colistimethate sodium. Recently, lower nephrotoxicity rates have been recorded even with polymyxin B. The suggested mechanism of renal dysfunction caused by polymyxin B is happened by increasing membrane permeability that leads to cell swelling due to the influx of water and ions that resulted in cell death. Additionally, fatty acid and amino acid components of polymyxin B are considered to be responsible for cell injury. Polymyxins nephrotoxicity is dose-dependent (Falagas \& Kasiakou, 2006). Briefly, recent studies of polymyxin B associated nephropathy is discussed below.

Ouderkirk et al. (20013) reported a 14\% prevalence of ARFin patients treated with polymyxin B $(\mathrm{n}=60)$. Those who developed ARF were older (mean age of 76 versus 59 years, $\mathrm{p}$ $=0.02)$. Higher mortality rate was reported $(57 \%$ versus $15 \%, \mathrm{p}<0.02)$ in ARF cases. Similarly, Holloway et al. (2006) reportedARFin $21.2 \%(\mathrm{n}=7 / 33)$ patients. None of the ARFrequired dialysis and creatinine levels returned to the normal range with discontinuation of polymyxin B in $71.4 \%(n=5 / 7)$ cases. Furtado et al. (2007) reported nephrotoxicity in9.4\% of patients with $P$. aeruginosa associated nosocomial pneumonia treated with polymyxin B. Also, there was no difference in ARF occurrence in patients who had favorable or unfavorable results.

Few studies were performed to recognize the factors associated with renal dysfunction due to polymyxin B. Bahlis et al. (2015) in a retrospective cohort study identified $43 \%$ of patients of renal injury by RIFLE (Risk, Injury, and Failure; Loss; and end-stage kidney disease) criteria. They observed hypotension (OR 2.79; $\mathrm{p}=0.006$ ) and concomitant vancomycin use (OR 2.79; $\mathrm{p}=00.011)$ as independent predictors of renal injury. Similarly, Dubrovskaya et al. (2015) in a retrospective cohort study evaluated 192 patients who received polymyxin B for more than 72 hours. In a mean duration of 9.5 days of treatment, the renal injury was found in $45.8 \%$ of patients. They reported daily dose based on actual weight (hazard ratio (HR) 1.73, p = 0.022), concomitant vancomycin (HR 1.89, $\mathrm{p}=0.005$ ), and use of contrast media (HR 1.79, $\mathrm{p}=0.009$ ) as independent risk factors for nephrotoxicity. In another multicenter, retrospective cohort study, a comparison of nephrotoxicity rates between colistimethate sodium ( $\mathrm{n}=121)$ and polymyxin B $(\mathrm{n}=104)$ was tried by Phe et al. $(2014)$ to validate their findings of in vitro cytotoxicity study. Patients administrating polymyxin $\mathrm{B}$ for more than 72 hours who had normal kidney function were assessed.

In risk factors matched analysis, observed rates of nephrotoxicity were significantly ( $\mathrm{p}=$ 0.004) higher with colistimethate sodium (55.3\%) in comparison to polymyxin B (21.1\%). On a multivariate analysis, significant and independent association of renal toxicity due to colistimethate was recorded with age (OR: 1.04, 95\% CI, 1.00, 1.07), treatment duration (OR: $1.08,95 \% \mathrm{CI}, 1.02,1.15)$, and daily dose based on body weight (OR: 1.40, 95\% CI, 1.05, 1.88). A prospective comparison between two polymyxins is required for further substantiation of this finding. A prospective cohort evaluation from Rigatto et al. (2015) in410 patients 
administrating polymyxin B for more than 48 hours reported acute renal toxicity in $46.1 \%$ of cases. Dose of polymyxin $\geq 150 \mathrm{mg} /$ day was significantly associated with renal dysfunction (HR 1.95, p = 0.01). It is of interest to show that the increased risk was maximal for dose range from 150 to $199 \mathrm{mg} /$ day and no further significant increase was observed for even higher doses. They found renal injury as an independent predictor of 30-day mortality (HR 1.35, p = 0.06) while the dose over $150 \mathrm{mg} /$ day did not increase mortality. This paradox calls for careful patient assessment. A higher dose may be associated with mortality but simultaneous renal dysfunction is increased. Exploring the underlying predisposing factors such as hypotension, use of vancomycin, or any contrast media is necessary. Modification of these abnormalities might assist in decreasing the incidence of renal injury with polymyxin B. In this scope, Rigatto et al. (2016) (Falagas \& Kasiakou, 2006). Studied mortality outcomes in patients of renal replacement therapy (RRT). In 88 RRT patients administrating polymyxin B (1.5 to 3 $\mathrm{mg} / \mathrm{kg} /$ day) for more than 48 hours, 30-day mortality was $51.1 \%$. A daily dose above $200 \mathrm{mg}$ was associated with lower mortality (HR $0.35, \mathrm{n}=0.03$ ). Therefore, a higher dose is effective in lowering mortality even in RRTcases.

\section{Neurotoxicity}

The incidence of neuropathy because of polymyxins administration is about $7 \%$. Its symptoms are similar to any other neuropathy including weakness, paraesthesia, ophthalmoplegia, dysphagia, ataxia, and neuromuscular weakness sometimes resulting in respiratory failure. Colistin/colistimethate sodium are mostly induced neurotoxicity (Falagas $\&$ Kasiakou, 2006). However, no severe forms of neurotoxicity necessitating respiratory support have been recorded in the last twenty years (Zavascki et al. (2006) identified one case of new-onset altered mental status and one with distal paraesthesia.

Neuropathy manifesting as seizures and neuromuscular weakness which were possibly due to polymyxin B in two (7\%) cases was studied bySobieszczyk et al. (2004). Recently two cases of polymyxin B-induced neuropathy were reported by Weinstein et al. (2009). The first case was a 60-year-old obese diabetic female with other multiple ailments and was on treatment with multiple medications including varenicline and quetiapine. Polymyxin B loading dose was $20000 \mathrm{U} / \mathrm{Kg}$ in two divided doses. It was initiated for K. Pneumonia identified in urine culture that was only sensitive to polymyxin B. She developed oral paraesthesia within 1 hour of starting IV infusion. The second patient was a 57-year-old male having ascending cholangitis. MDR $K$. pneumonia susceptible only to polymyxin B, gentamicin, and trimethoprimsulfamethoxazole was found in drain fluid culture. Multiple medications were prescribed during hospitalization. For pancreatic abscess, the patient was advised with 30-day treatment with polymyxin B and imipenem cilastatin. After 30 days, oral and lower extremity paraesthesia were occurring but symptoms reversed with discontinuation of polymyxin $\mathrm{B}$. There was no rechallenge attempted in either case. Although it is not commonly reported, caution is necessary with increasing doses of polymyxin B for monitoring neurotoxicity.

\section{Congenital Anomalies}

It is rarely reported with polymyxin Kazy et al. (2005) recorded crude OR of 0.8 for the first trimester. Anomalies involving cardiovascular malformations, neural tube defect, 
microcephaly, limb reduction defect, and congenital talipes equinovarus are recorded. Because of the small number of cases, the risk appears small though existent. In general, there is limited data for polymyxin B and assessment in a larger sample is important to confirm the causal effect (Zavascki et al., 2007).

\section{Tolerability of polymyxin B}

In general, polymyxin B is well tolerated (Zavascki, (2011). Milder side effects may include rash, pruritus, dermatitis, and fever. They are probably the result of the histaminereleasing action of polymyxin B (Zavascki et al., 2007).

\section{Dosage and Administration of Polymyxin B}

The recommended daily dose of polymyxin B is 1.5 to $2.5 \mathrm{mg} / \mathrm{kg}$ in common. It is administered IV in two divided doses as a one-hour infusion. This dose is well-tolerated in an empirical setting (Zavascki et al., 2007). Some studies suggest a dose of up to $3 \mathrm{mg} / \mathrm{kg} / \mathrm{day}$ being used in a clinical setting (Sandri et al., 2013; Zavascki et al., 2008)). Evidence suggests that a daily dose of $200 \mathrm{mg}$ and above is associated with better mortality outcomes as we mention above, however, the renal injury requires to be cautiously monitored at such higher dose. Therefore, doses above $3 \mathrm{mg} / \mathrm{kg} /$ day cannot be recommended for safety concerns (Sandri et al., 2013). Evaluation of baseline renal function may be important but dosing is not affected by renal function as polymyxin B is majorly eliminated by non-renal mechanisms. Prescribing polymyxin $\mathrm{B}$ in an adequate dosage is essential to avoid underdosing in lieu of renal dysfunction (Zavascki, 2011; Kwa et al., 2011).

\section{Positioning polymyxins in therapy}

Recently, polymyxin B has reemerged in a clinical setting. Its use is likely to continue to increase since new drugs for the treatment of infections caused by MDR Gramnegative bacteria are beyond a distant horizon. Therefore, we can consider polymyxin B as the last resort therapy for MDR and XDR Gram-negative infections particularly those caused by $K$. pneumoniae, A. baumannii, and P. aeruginosa. PK data have made understanding of polymyxin B kinetics more clear. Also, it helps explore dosing regimens. Dosing based on actual body weight is helpful and should not be based on renal function. Efficacy against superbugs producing NDM-1 beta-lactamases makes polymyxin B crucial in infection management. IV administration has been most effective in improving clinical, microbiological, and mortality outcomes not only in adults but in critically ill children also. Initial dose selection and titration are simple and more predictable for polymyxin B because of smaller interindividual variability and lack of impact of renal function on drug clearance. Therapeutic drug monitoring for polymyxin B lacks the significant difficulties that exist for colistin (Nation et al., 2014).

\section{Conclusion}

Maintaining polymyxins efficiency in the era of resistant superbugs is a critical and vital issue to extend its clinical use. Currently, polymyxins are the last resort for most MDR 
and/or XDR Gram-negative infections including A. baumannii, $K$. pneumoniae and $P$. aeruginosa. Thus understanding of polymyxins PK and PD is a very important issue for optimization of their usage. Validated or even empiric combinations of polymyxins with other antibiotics is recommended for avoiding treatment failure, reduce infection-related mortality and prevent the emergence of resistance.

\section{References}

Abdul Rahim, N., Cheah, S. E., Johnson, M. D., Yu, H., Sidjabat, H. E., Boyce, J., ... \& Li, J. (2015). Synergistic killing of NDM-producing MDR Klebsiella pneumoniae by two 'old'antibiotics-polymyxin B and chloramphenicol. Journal of Antimicrobial Chemotherapy, 70(9), 2589-2597.

Bahlis, L. F., Diogo, L. P., Lemons, D., \& Klaus, D. (2015). Risk factors for acute kidney injury in patients treated with polymyxin B at a Tertiary Care Medical Center. Brazilian Journal of Nephrology, 37, 446-450.

Barth, N., Ribeiro, V. B., \& Zavascki, A. P. (2015). In vitro activity of polymyxin B plus imipenem, meropenem, or tigecycline against KPC-2-producing Enterobacteriaceae with high MICs for these antimicrobials. Antimicrobial agents and chemotherapy, 59(6), 3596-3597.

Ben-Ami, R., Lewis, R. E., Tarrand, J., Leventakos, K., \& Kontoyiannis, D. P. (2010). Antifungal activity of colistin against Mucorales species in vitro and in a murine model of Rhizopus oryzae pulmonary infection. Antimicrobial agents and chemotherapy, 54(1), 484-490.

Berditsch, M., Jäger, T., Strempel, N., Schwartz, T., Overhage, J., \& Ulrich, A. S. (2015). Synergistic effect of membrane-active peptides polymyxin B and gramicidin $S$ on multidrug-resistant strains and biofilms of Pseudomonas aeruginosa. Antimicrobial agents and chemotherapy, 59(9), 5288-5296.

Bergen, P. J., Bulman, Z. P., Saju, S., Bulitta, J. B., Landersdorfer, C., Forrest, A., ... \& Tsuji, B. T. (2015). Polymyxin combinations: pharmacokinetics and pharmacodynamics for rationale use. Pharmacotherapy: The Journal of Human Pharmacology and Drug Therapy, 35(1), 34-42.

Bergen, P. J., Li, J., Nation, R. L., Turnidge, J. D., Coulthard, K., \& Milne, R. W. (2008). Comparison of once-, twice-and thrice-daily dosing of colistin on antibacterial effect and emergence of resistance: studies with Pseudomonas aeruginosa in an in vitro pharmacodynamic model. Journal of Antimicrobial Chemotherapy, 61(3), 636-642.

Bowers, D. R., Cao, H., Zhou, J., Ledesma, K. R., Sun, D., Lomovskaya, O., \& Tam, V. H. (2015). Assessment of minocycline and polymyxin B combination against Acinetobacter baumannii. Antimicrobial agents and chemotherapy, 59(5), 2720-2725.

C. de la Fuente-Nu'nez, F. Re ffuveille, E. F. Haney,S. K. Straus and R. E. Hancock, PLoSPathog., vol. 10,e1004152, 2014.

Cai, B., Cai, Y., Liew, Y. X., Chua, N. G., Teo, J. Q. M., Lim, T. P., ... \& Kwa, A. L. H. (2016). Clinical efficacy of polymyxin monotherapy versus nonvalidated polymyxin combination therapy versus validated polymyxin combination therapy in extensively 
drug-resistant Gram-negative bacillus infections. Antimicrobial agents and chemotherapy, 60(7), 4013-4022.

Crusio, R., Rao, S., Changawala, N., Paul, V., Tiu, C., van Ginkel, J., ... \& Kupfer, Y. (2014). Epidemiology and outcome of infections with carbapenem-resistant Gram-negative bacteria treated with polymyxin B-based combination therapy. Scandinavian journal of infectious diseases, 46(1), 1-8.

Deris, Z. Z., Akter, J., Sivanesan, S., Roberts, K. D., Thompson, P. E., Nation, R. L., ... \& Velkov, T. (2014). A secondary mode of action of polymyxins against Gram-negative bacteria involves the inhibition of $\mathrm{NADH}$-quinone oxidoreductase activity. The Journal of antibiotics, 67(2), 147-151.

Dubrovskaya, Y., Chen, T. Y., Scipione, M. R., Esaian, D., Phillips, M. S., Papadopoulos, J., \& Mehta, S. A. (2013). Risk factors for treatment failure of polymyxin B monotherapy for carbapenem-resistant Klebsiella pneumoniae infections. Antimicrobial agents and chemotherapy, 57(11), 5394-5397.

Dubrovskaya, Y., Prasad, N., Lee, Y., Esaian, D., Figueroa, D. A., \& Tam, V. H. (2015). Risk factors for nephrotoxicity onset associated with polymyxin B therapy. Journal of Antimicrobial Chemotherapy, 70(6), 1903-1907.

Elias, L. S., Konzen, D., Krebs, J. M., \& Zavascki, A. P. (2010). The impact of polymyxin B dosage on in-hospital mortality of patients treated with this antibiotic. Journal of antimicrobial chemotherapy, 65(10), 2231-2237.

Falagas, M. E., \& Kasiakou, S. K. (2006). Toxicity of polymyxins: a systematic review of the evidence from old and recent studies. Critical care, 10(1), 1-13.

Falagas, M. E., Kasiakou, S. K., \& Saravolatz, L. D. (2005). Colistin: the revival of polymyxins for the management of multidrug-resistant gram-negative bacterial infections. Clinical infectious diseases, 40(9), 1333-1341.

Furtado, G. H. C., d'Azevedo, P. A., Santos, A. F., Gales, A. C., Pignatari, A. C. C., \& Medeiros, E. A. S. (2007). Intravenous polymyxin B for the treatment of nosocomial pneumonia caused by multidrug-resistant Pseudomonas aeruginosa. International journal of antimicrobial agents, 30(4), 315-319.

Gopal, R., Kim, Y. G., Lee, J. H., Lee, S. K., Chae, J. D., Son, B. K., ... \& Park, Y. (2014). Synergistic effects and antibiofilm properties of chimeric peptides against multidrugresistant Acinetobacter baumannii strains. Antimicrobial agents and chemotherapy, 58(3), 1622-1629.

Groisman, E. A., Kayser, J., \& Soncini, F. C. (1997). Regulation of polymyxin resistance and adaptation to low-Mg2+ environments. Journal of bacteriology, 179(22), 7040-7045.

Gupta, S., Govil, D., Kakar, P. N., Prakash, O., Arora, D., Das, S., ... \& Malhotra, A. (2009). Colistin and polymyxin B: a re-emergence. Indian journal of critical care medicine: peer-reviewed, official publication of Indian Society of Critical Care Medicine, 13(2), 49.

Hagihara, M., Housman, S. T., Nicolau, D. P., \& Kuti, J. L. (2014). In vitro pharmacodynamics of polymyxin B and tigecycline alone and in combination against carbapenemresistant Acinetobacter baumannii. Antimicrobial agents and chemotherapy, 58(2), 874-879. 
Holloway, K. P., Rouphael, N. G., Wells, J. B., King, M. D., \& Blumberg, H. M. (2006). Polymyxin B and doxycycline use in patients with multidrug-resistant Acinetobacter baumannii infections in the intensive care unit. Annals of Pharmacotherapy, 40(11), 1939-1945.

Hooton, T. M., \& Levy, S. B. (2001). Antimicrobial Resistence: A Plan of Action for Community Practice. American family physician, 63(6), 1087.

Hsu, L. H., Wang, H. F., Sun, P. L., Hu, F. R., \& Chen, Y. L. (2017). The antibiotic polymyxin $\mathrm{B}$ exhibits novel antifungal activity against Fusarium species. International journal of antimicrobial agents, 49(6), 740-748.

I. d'Angelo, B. Casciaro, A. Miro, F. Quaglia, M. L. Mangoniand F. Ungaro, Colloids Surf., B, vol. 35, pp. 717-725, 2015.

Kazy, Z., Puho, E., \& Czeizel, A. E. (2005). Parenteral polymyxin B treatment during pregnancy. Reproductive toxicology (Elmsford, NY), 20(2), 181-182.

Kvitko, C. H., Rigatto, M. H., Moro, A. L., \& Zavascki, A. P. (2011). Polymyxin B versus other antimicrobials for the treatment of Pseudomonas aeruginosa bacteraemia. Journal of Antimicrobial Chemotherapy, 66(1), 175-179.

Kwa, A. L., Abdelraouf, K., Low, J. G., \& Tam, V. H. (2011). Pharmacokinetics of polymyxin $\mathrm{B}$ in a patient with renal insufficiency: a case report. Clinical Infectious Diseases, 52(10), 1280-1281.

Kwa, A. L., Lim, T. P., Low, J. G., Hou, J., Kurup, A., Prince, R. A., \& Tam, V. H. (2008). Pharmacokinetics of polymyxin B1 in patients with multidrug-resistant Gramnegative bacterial infections. Diagnostic microbiology and infectious disease, 60(2), 163-167.

Kwa, A. L., Tam, V. H., \& Falagas, M. E. (2008). Polymyxins: a review of the current status including recent developments. Ann Acad Med Singapore, 37(10), 870-883.

Landman, D., Georgescu, C., Martin, D. A., \& Quale, J. (2008). Polymyxins revisited. Clinical microbiology reviews, 21(3), 449-465.

Lim, T. P., Tan, T. Y., Lee, W., Sasikala, S., Tan, T. T., Hsu, L. Y., \& Kwa, A. L. (2011). Invitro activity of polymyxin $\mathrm{B}$, rifampicin, tigecycline alone and in combination against carbapenem-resistant Acinetobacter baumannii in Singapore. PloS one, 6(4), e18485.

Lora-Tamayo, J., Murillo, O., Bergen, P. J., Nation, R. L., Poudyal, A., Luo, X., ... \& Li, J. (2014). Activity of colistin combined with doripenem at clinically relevant concentrations against multidrug-resistant Pseudomonas aeruginosa in an in vitro dynamic biofilm model. Journal of antimicrobial chemotherapy, 69(9), 2434-2442.

McLeod, G. I., \& Spector, M. P. (1996). Starvation-and Stationary-phase-induced resistance to the antimicrobial peptide polymyxin B in Salmonella typhimurium is RpoS (sigma (S)) independent and occurs through both phoP-dependent and-independent pathways. Journal of Bacteriology, 178(13), 3683-3688.

Nation, R. L., Li, J., Cars, O., Couet, W., Dudley, M. N., Kaye, K. S., ... \& Turnidge, J. D. (2015). Framework for optimisation of the clinical use of colistin and polymyxin B: the Prato polymyxin consensus. The Lancet infectious diseases, 15(2), 225-234.

Nation, R. L., Velkov, T., \& Li, J. (2014). Colistin and polymyxin B: peas in a pod, or chalk and cheese?. Clinical infectious diseases, 59(1), 88-94. 
Nelson, B. C., Eiras, D. P., Gomez-Simmonds, A., Loo, A. S., Satlin, M. J., Jenkins, S. G., ... \& Kubin, C. J. (2015). Clinical outcomes associated with polymyxin B dose in patients with bloodstream infections due to carbapenem-resistant Gram-negative rods. Antimicrobial agents and chemotherapy, 59(11), 7000-7006.

Ouderkirk, J. P., Nord, J. A., Turett, G. S., \& Kislak, J. W. (2003). Polymyxin B nephrotoxicity and efficacy against nosocomial infections caused by multiresistant gram-negative bacteria. Antimicrobial agents and chemotherapy, 47(8), 2659-2662.

Pankey, G., Ashcraft, D., Kahn, H., \& Ismail, A. (2014). Time-kill assay and Etest evaluation for synergy with polymyxin $\mathrm{B}$ and fluconazole against Candida glabrata. Antimicrobial agents and chemotherapy, 58(10), 5795-5800.

Phe, K., Lee, Y., McDaneld, P. M., Prasad, N., Yin, T., Figueroa, D. A., ... \& Tam, V. H. (2014). In vitro assessment and multicenter cohort study of comparative nephrotoxicity rates associated with colistimethate versus polymyxin B therapy. Antimicrobial agents and chemotherapy, 58(5), 2740-2746.

Pietschmann, S., Hoffmann, K., Voget, M., \& Pison, U. (2009). Synergistic effects of miconazole and polymyxin B on microbial pathogens. Veterinary research communications, 33(6), 489.

Qamar, F. N., Jurair, H., \& Haque, A. (2014). Multi-drug resistant gram negative infections and use of intravenous polymyxin B in critically ill children of developing country: retrospective cohort study. BMC infectious diseases, 14(1), 1-7.

R. Rosales-Reyes, M. D. Alc’antara-Curiel, M. D.JarilloQuijada, C. Gayosso-V’azquez, M. Mor $\square$ n-Otero,E.Rodr'́guez-Noriega and J. Santos-Preciado, Chemotherapy, vol. 61, pp. 8-14, 2015-2016.

Rigatto, M. H., Behle, T. F., Falci, D. R., Freitas, T., Lopes, N. T., Nunes, M., ... \& Zavascki, A. P. (2015). Risk factors for acute kidney injury (AKI) in patients treated with polymyxin B and influence of AKI on mortality: a multicentre prospective cohort study. Journal of Antimicrobial Chemotherapy, 70(5), 1552-1557.

Rigatto, M. H., Falci, D. R., Lopes, N. T., \& Zavascki, A. P. (2016). Clinical features and mortality of patients on renal replacement therapy receiving polymyxin B. International journal of antimicrobial agents, 47(2), 146-150.

Rigatto, M. H., Vieira, F. J., Antochevis, L. C., Behle, T. F., Lopes, N. T., \& Zavascki, A. P. (2015). Polymyxin B in combination with antimicrobials lacking in vitro activity versus polymyxin B in monotherapy in critically ill patients with Acinetobacter baumannii or Pseudomonas aeruginosa infections. Antimicrobial agents and chemotherapy, 59(10), 6575-6580.

Rosales-Reyes, R., Alcántar-Curiel, M. D., Jarillo-Quijada, M. D., Gayosso-Vázquez, C., del Rayo Morfin-Otero, M., Rodríguez-Noriega, E., \& Santos-Preciado, J. I. (2016). Biofilm formation and susceptibility to polymyxin B by a highly prevalent clone of multidrug-resistant Acinetobacter baumannii from a Mexican Tertiary Care Hospital. Chemotherapy, 61(1), 8-14.

Sandri, A. M., Landersdorfer, C. B., Jacob, J., Boniatti, M. M., Dalarosa, M. G., Falci, D. R., ... \& Zavascki, A. P. (2013). Population pharmacokinetics of intravenous polymyxin 
B in critically ill patients: implications for selection of dosage regimens. Clinical infectious diseases, 57(4), 524-531.

Schemuth, H., Dittmer, S., Lackner, M., Sedlacek, L., Hamprecht, A., Steinmann, E., ... \& Steinmann, J. (2013). In vitro activity of colistin as single agent and in combination with antifungals against filamentous fungi occurring in patients with cystic fibrosis. Mycoses, 56(3), 297-303.

Schwartz, S. N., Medoff, G., Kobayashi, G. S., Kwan, C. N., \& Schlessinger, D. (1972). Antifungal properties of polymyxin $\mathrm{B}$ and its potentiation of tetracycline as an antifungal agent. Antimicrobial agents and chemotherapy, 2(1), 36-40.

See comment in PubMed Commons below E. K. Adams,D. S.Ashcratand G. A. Pankey, Am. J. Med. Sci., vol. 35, pp.265-270, 2016.

Shaikh, S., Fatima, J., Shakil, S., Rizvi, S. M. D., \& Kamal, M. A. (2015). Antibiotic resistance and extended spectrum beta-lactamases: Types, epidemiology and treatment. Saudi journal of biological sciences, 22(1), 90-101..

Sobieszczyk, M. E., Furuya, E. Y., Hay, C. M., Pancholi, P., Della-Latta, P., Hammer, S. M., \& Kubin, C. J. (2004). Combination therapy with polymyxin B for the treatment of multidrug-resistant Gram-negative respiratory tract infections. Journal of Antimicrobial Chemotherapy, 54(2), 566-569.

Tam, V. H., Cao, H., Ledesma, K. R., \& Hu, M. (2011). In vitro potency of various polymyxin B components. Antimicrobial agents and chemotherapy, 55(9), 4490-4491.

Tam, V. H., Schilling, A. N., Vo, G., Kabbara, S., Kwa, A. L., Wiederhold, N. P., \& Lewis, R. E. (2005). Pharmacodynamics of polymyxin B against Pseudomonas aeruginosa. Antimicrobial agents and chemotherapy, 49(9), 3624-3630.

Tarquinio, K., Confreda, K., Shurko, J., \& LaPlante, K. (2014). Activities of tobramycin and polymyxin E against Pseudomonas aeruginosa biofilm-coated medical grade endotracheal tubes. Antimicrobial agents and chemotherapy, 58(3), 1723-1729.

Teo, J., Lim, T. P., Hsu, L. Y., Tan, T. Y., Sasikala, S., Hon, P. Y., ... \& Apisarnthanarak, A. (2015). Extensively drug-resistant Acinetobacter baumannii in a Thai hospital: a molecular epidemiologic analysis and identification of bactericidal Polymyxin Bbased combinations. Antimicrobial resistance and infection control, 4(1), 1-7.

Thamlikitkul, V., Tiengrim, S., \& Seenama, C. (2014). In vitro activity of polymyxin B against carbapenem-resistant Acinetobacter baumannii. J Med Assoc Thai, 97(12), 1254-8.

Theuretzbacher, U., Van Bambeke, F., Cantón, R., Giske, C. G., Mouton, J. W., Nation, R. L., ... \& Kahlmeter, G. (2015). Reviving old antibiotics. Journal of Antimicrobial Chemotherapy, 70(8), 2177-2181.

Ventola, C. L. (2015). The antibiotic resistance crisis: part 1: causes and threats. Pharmacy and therapeutics, 40(4), 277.

Venturini, T. P., Rossato, L., Chassot, F., Keller, J. T., Piasentin, F. B., Santurio, J. M., \& Alves, S. H. (2016). In vitro synergistic combinations of pentamidine, polymyxin B, tigecycline and tobramycin with antifungal agents against Fusarium spp. Journal of medical microbiology, 65(8), 770-774.

Wei, W., Yang, H., Hu, L., Ye, Y., \& Li, J. (2015). Activity of levofloxacin in combination with. Journal of Microbiology, Immunology and Infection, 20, 1e10. 
Weinstein, L., Doan, T. L., \& Smith, M. A. (2009). Neurotoxicity in patients treated with intravenous polymyxin B: Two case reports. American Journal of Health-System Pharmacy, 66(4), 345-347.

Zavascki, A. P. (2011). Dose adjustment of polymyxins for renal insufficiency. Antimicrobial agents and chemotherapy, 55(10), 4940-4940.

Zavascki, A. P., Goldani, L. Z., Cao, G., Superti, S. V., Lutz, L., Barth, A. L., ... \& Li, J. (2008). Pharmacokinetics of intravenous polymyxin $\mathrm{B}$ in critically ill patients. Clinical infectious diseases, 47(10), 1298-1304.

Zavascki, A. P., Goldani, L. Z., Li, J., \& Nation, R. L. (2007). Polymyxin B for the treatment of multidrug-resistant pathogens: a critical review. Journal of antimicrobial chemotherapy, 60(6), 1206-1215.

Zeidler, U., Bougnoux, M. E., Lupan, A., Helynck, O., Doyen, A., Garcia, Z., ... \& d'Enfert, C. (2013). Synergy of the antibiotic colistin with echinocandin antifungals in Candida species. Journal of Antimicrobial Chemotherapy, 68(6), 1285-1296,

Zhai, B., \& Lin, X. (2013). Evaluation of the anticryptococcal activity of the antibiotic polymyxin B in vitro and in vivo. International journal of antimicrobial agents, 41(3), 250-254.

Zhai, B., Zhou, H., Yang, L., Zhang, J., Jung, K., Giam, C. Z., ... \& Lin, X. (2010). Polymyxin $\mathrm{B}$, in combination with fluconazole, exerts a potent fungicidal effect. Journal of Antimicrobial Chemotherapy, 65(5), 931-938. 